\title{
Perforated Block Copolymer Vesicles with A Highly Folded Membrane
}

Yongming Chen, * Jianzhong Du, Ming Xiong, and Hongxia Guo*.

State Key Laboratory of Polymer Physics and Chemistry, Joint Laboratory of Polymer Science and Materials, Institute of Chemistry, The Chinese Academy of Sciences, Beijing 100080, P. R. China.

\section{Hiroshi Jinnai* and Takeshi Kaneko}

Department of Macromolecular Science and Engineering, Graduate School of Science and Engineering, Kyoto Institute of Technology, Goshokaido-cho, Matsugasaki, Kyoto 606-8585, Japan.

\section{Supporting Information}

\section{Experimental Section}

The block copolymer, $\mathrm{PEO}_{45}-b$-PTMSPMA 180 (the composition was evaluated by ${ }^{1} \mathrm{H}$ NMR spectrum in $\mathrm{CDCl}_{3}, M_{\mathrm{w}} / M_{\mathrm{n}}$ was 1.34 given by $\mathrm{SEC}$ calibrated by PS standard), was synthesized by atom transfer radical polymerization of TMSPMA initiated with monomethoxy poly(ethylene oxide) 2-bromoisobutyrate [Du, J. Z.; Chen, Y. M. Macromolecules 2004, 37, 6322]. To induce aggregate, the copolymer $(1 \mathrm{mg})$ was dissolved in methanol $(1.0 \mathrm{~mL})$, and then water was added dropwise within 10 min by a syringe under vigorous stirring. A colloid solution with $39 \mathrm{wt} \%$ of water content was given and appearance of cloudy solution indicated the aggregate formation. After five hours, TEA (20 $\mu \mathrm{L})$ was added to induce the hydrolysis and cross-linking reactions.

TEM images were obtained using a JEM 100 electron microscope. To prepare TEM samples, $5 \mu \mathrm{L}$ of vesicle solution was dropped on a carbon-coated copper grid and dried at ambient temperature. For observation of internal morphologies of the particles, a powder sample was embedded in mixture of epoxy resin and diethyltriamine $(100 / 11, \mathrm{~m} / \mathrm{m})$, and then placed an oven at $60{ }^{\circ} \mathrm{C}$ for $12 \mathrm{~h}$. The sections about 50 to $80 \mathrm{~nm}$ were obtained by microtoming. To obtain a SEM image, a drop of solution was spread on a silica wafer. Then the specimens were coated with platinum and viewed by a Hitachi S-4300 electron microscopy operated at $15 \mathrm{kV}$. The images were recorded by a digital camera. 
TEMT experiments were carried out on a JEM-2200FS (JEOL Ltd., Japan) operated at $200 \mathrm{kV}$. The digital data of the transmitted images were collected with a slow-scan CCD camera (Gatan USC1000, Gatan Inc.). Two sets of tilt series were taken at the same area of the specimen. A projection at each tilt angle was acquired with frame size of 1024 by 1024 pixels. To obtain achromatic projections, only the transmitted and elastically scattered electrons (electron energy loss: $0 \pm 30 \mathrm{eV}$ ) were selected by an energy filter installed in a JEM-2200FS ( $\Omega$ filter, JEOL Ltd., Japan). The tilt series were taken over the tilt angle from $-64^{\circ}$ to $+60^{\circ}$ with $1^{\circ}$ increment. The pixel size was $1.05 \mathrm{~nm}$.

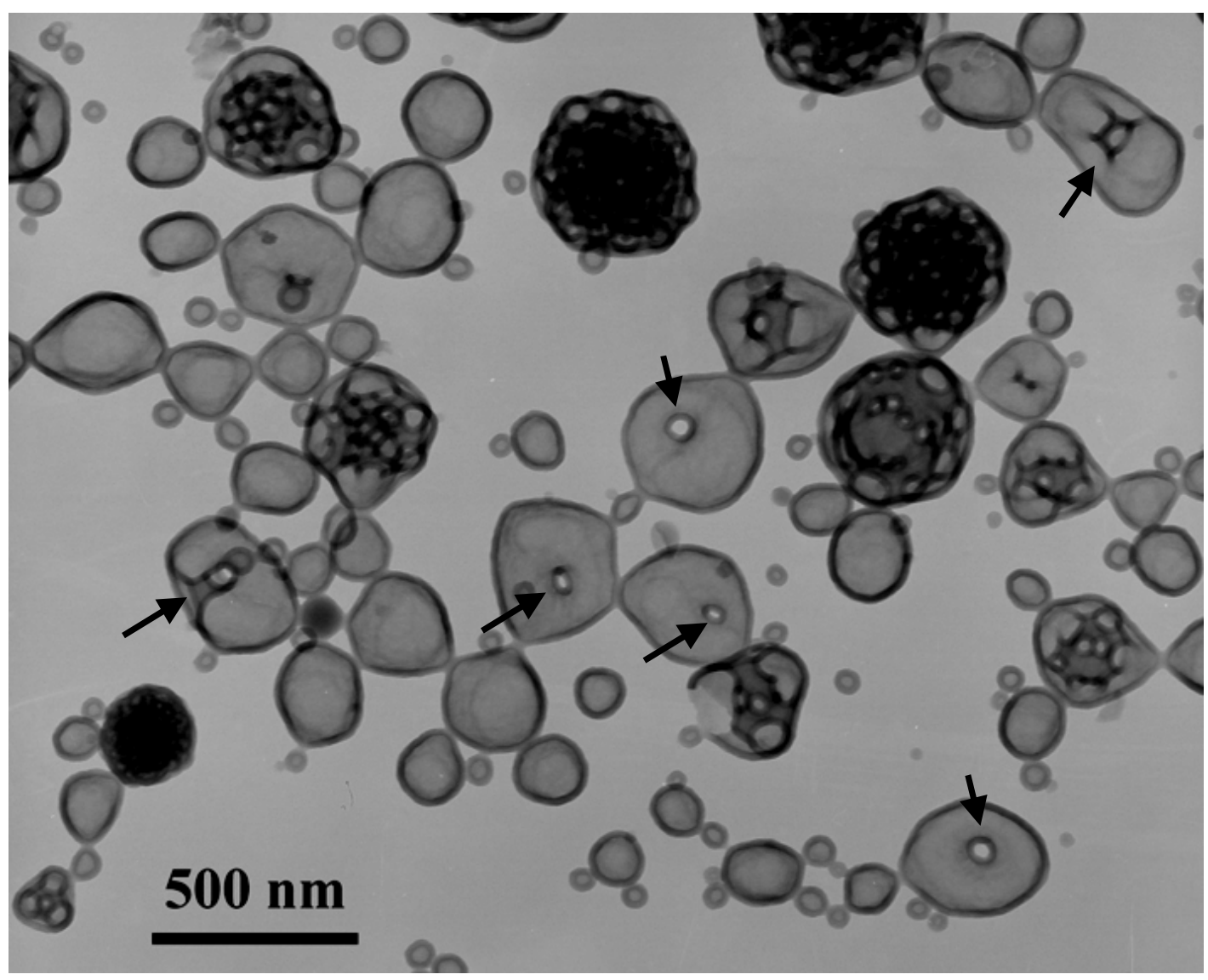

Figure S1. TEM image of the vesicles with one hole as pointed by the arrows. 


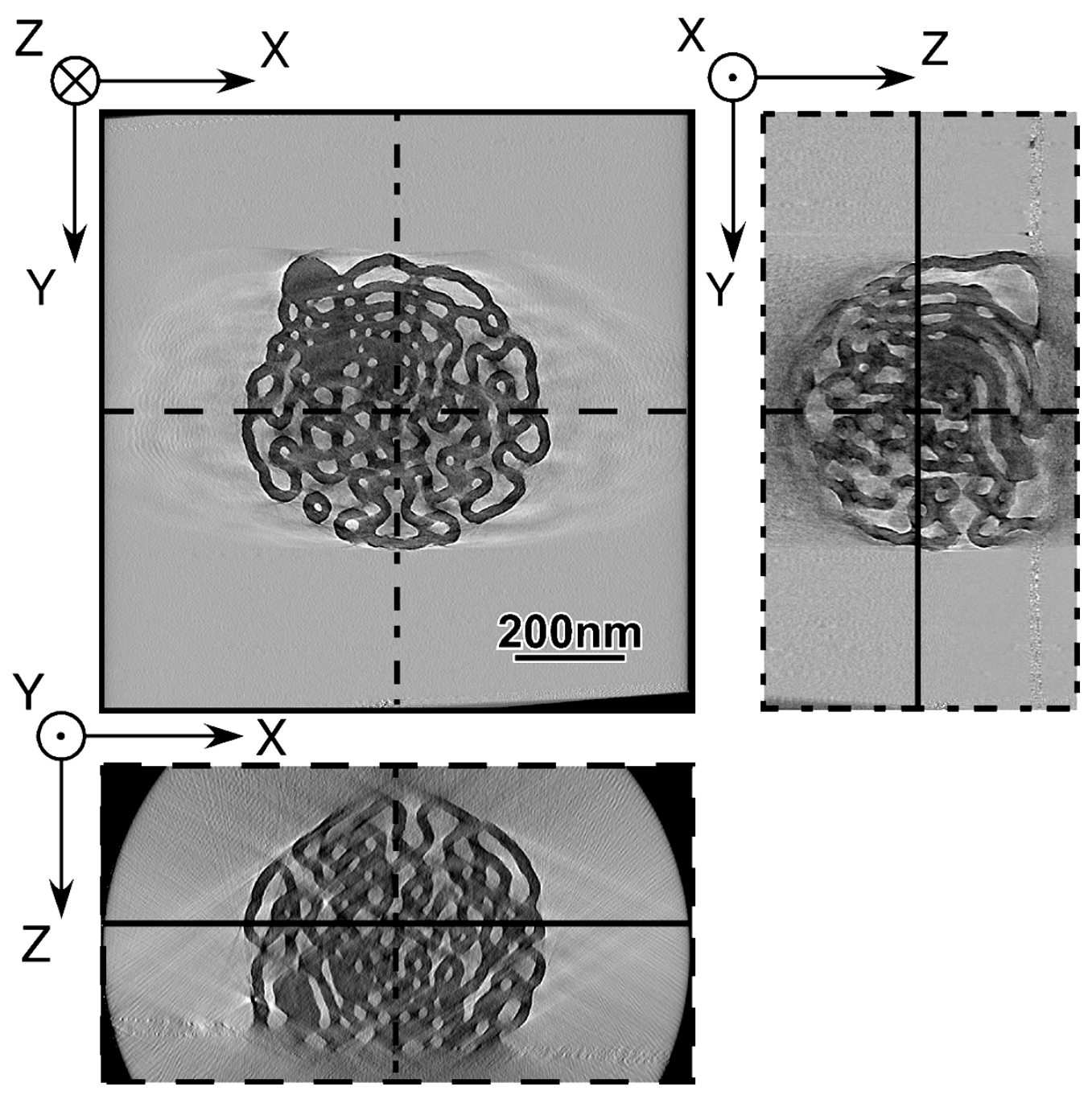

Figure S2. An orthogonal cross-sectional view of 3D reconstructions obtained from dual-axis tomography of one complex vesicle. 

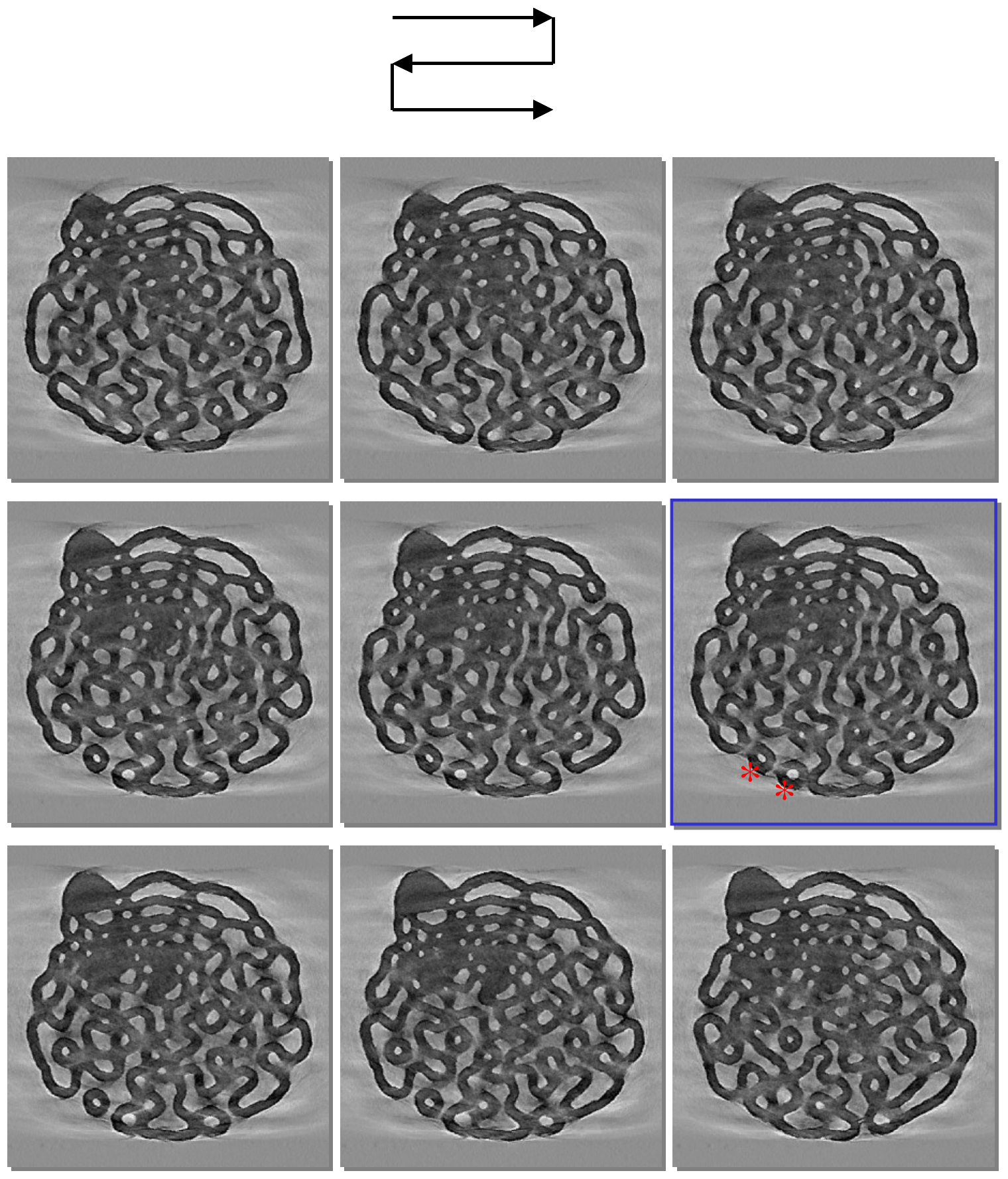

Figure S3. A series of TEM images of the adjacent slices from which one may learn how the tubes (red

*) formed and merged with neighboring spaces. Direction arrow shows the slices one-by-one adjacent to the slice (with blue frame) in Figure 3A. 